Quim. Nova, Vol. 35, No. 2, 262-267, 2012

\title{
ESTUDO COMPARATIVO DA CARACTERIZAÇÃO DE FILMES BIODEGRADÁVEIS DE AMIDO DE MANDIOCA CONTENDO POLPAS DE MANGA E DE ACEROLA
}

\author{
Carolina Oliveira de Souza*, Luciana Tosta Silva e Janice Izabel Druzian \\ Departamento de Análises Bromatológicas, Faculdade de Farmácia, Universidade Federal da Bahia, Rua Barão de Geremoabo, \\ s/n, 40171-970 Salvador - BA, Brasil
}

Recebido em 2/12/10; aceito em 3/7/11; publicado na web em 16/8/11

\begin{abstract}
COMPARATIVE STUDIES ON THE CHARACTERIZATION OF BIODEGRADABLE CASSAVA STARCH FILMS CONTAINING MANGO AND ACEROLA PULPS. Most compounds reinforcements have been used to improve thermals, mechanical and barrier properties of biopolymers films, whose performance is usually poor when compared to those of synthetic polymers. Biodegradables films have been developed by adding mango and acerola pulps in different concentrations $(0-17,1 \% \mathrm{w} / \mathrm{w})$ as antioxidants active compounds to cassava starch based biodegradable films. The effect of pulps was studied in terms of tensile properties, water vapor permeability, DSC, among other analysis of the films. The study demonstrated that the properties of cassava starch biodegradable films can be significantly altered through of incorporation mango and acerola pulps.
\end{abstract}

Keywords: bioactive films; natural additives; mechanical and thermal properties.

\section{INTRODUÇÃO}

As embalagens são utilizadas para preservar e proteger o produto embalado e, consequentemente, aumentar seu armazenamento. $\mathrm{O}$ uso de plásticos sintéticos como material de embalagem é grande, principalmente por sua disponibilidade, baixo custo e características funcionais, destacando-se as boas propriedades mecânicas, barreira aos gases e compostos aromáticos, e a facilidade de selagem térmica. ${ }^{1}$ No entanto, apesar das grandes vantagens seu uso crescente gera preocupação devido a problemas de contaminação ambiental decorrentes do descarte, uma vez que não são biodegradáveis e sua reciclagem consome grandes quantidades de energia térmica. $\mathrm{O}$ interesse de manter, ou melhorar, a qualidade dos produtos embalados e, ao mesmo tempo, reduzir o desperdício de embalagens, tem encorajado a exploração de novos materiais de embalagens, como os filmes biodegradáveis formulados com matérias-primas oriundas de recursos renováveis. ${ }^{2}$ Entretanto, o uso comercial de filmes comestíveis formulados a partir de matrizes biodegradáveis tem sido limitado por causa de problemas relacionados às propriedades mecânicas usualmente pobres e fraca barreira à umidade, quando comparados aos polímeros sintéticos.

Diferentes compósitos têm sido desenvolvidos por adição de compostos de reforço a filmes de polímeros biodegradáveis, buscando melhorar as propriedades térmicas, mecânicas e de barreira. Entretanto, muitas vezes, muitos destes materiais de reforço resultam numa pior adesão na interface com outros componentes da matriz. ${ }^{3}$

Diferentes compostos também têm sido incorporados aos biofilmes, visando conferir-lhes propriedades de barreiras específicas. Um dos principais sistemas são os biofilmes com ação antioxidante, que retardam ou diminuem o processo de oxidação do produto embalado, ${ }^{4}$ e são de grande importância para a indústria, principalmente dos ramos alimentício e farmacêutico. Tradicionalmente, as embalagens ativas antioxidantes existentes no mercado utilizam antioxidantes sintéticos e matrizes poliméricas não biodegradáveis em sua formulação. ${ }^{5}$ Como alternativa têm surgido filmes biodegradáveis ativos que podem ser obtidos a partir de fontes renováveis, filmes flexíveis formulados a partir de fécula de mandioca podem ser investigados como matriz

\footnotetext{
*e-mail: carolinaods@hotmail.com
}

para incorporação de agentes antioxidantes. ${ }^{6,7}$ No entanto, ao se fazer a incorporação de antioxidantes naturais a filmes biodegradáveis, ocorrem simultaneamente alterações das propriedades mecânicas, térmicas e de permeabilidade ao vapor de água do novos compósitos.

A manga (Mangifera indica L.), a acerola (Malpighia emarginata D.C.) e seus derivados contêm concentrações elevadas de antioxidantes como carotenoides e compostos fenólicos, que podem ser incorporados a filmes biodegradáveis como compostos ativos. Estas frutas contêm também quantidades significativas de fibras, especialmente pectina, amido e derivados de celulose ${ }^{8,9}$ podendo, dessa forma, contribuir simultaneamente como aditivo para reforço mecânico e térmico, melhorando a barreira à água dos biofilmes, além da ação antioxidante decorrente dos pigmentos e outros compostos. A dispersão uniforme destes aditivos na interface da matriz resulta em mudanças na mobilidade molecular, na relaxação e, consequentemente, nas propriedades mecânicas e térmicas do compósito resultante. Compostos com grandes raios moleculares, como fibras, são particularmente interessantes devido à maior superfície de contato, e podem promover o reforço desejado. ${ }^{10,11}$

As propriedades mecânicas dos biofilmes são características determinantes para a sua utilização como material de embalagem, devido ao manuseio a que estão sujeitos os produtos durante sua distribuição e comercialização. As propriedades funcionais dos biofilmes são fortemente influenciadas pelas condições de processos, pela formulação e pelos plastificantes usados. As propriedades requeridas para os biofilmes dependem principalmente das características do produto que será embalado, entretanto, baixa permeabilidade ao oxigênio é requerida em produtos sensíveis à oxidação, como gorduras poli-insaturadas.

O emprego do amido na elaboração de biofilmes apresenta como vantagem a biodegrabilidade, no entanto, suas propriedades são inferiores aos plásticos convencionais, formando materiais quebradiços e higroscópicos. Muitos trabalhos têm sido publicados utilizando amidos modificados, blendas de amido e polímeros sintéticos ou compósitos de amido com fibras vegetais, visando melhorar as propriedades mecânicas dos materiais preparados. ${ }^{12-14} \mathrm{O}$ objetivo do presente estudo foi avaliar o efeito da incorporação de diferentes teores de polpas de manga e de acerola como aditivos em biofilmes formulados com fécula de mandioca através da determinação das 
propriedades físico-químicas, térmicas e mecânicas dos mesmos. Um biofilme de fécula de mandioca sem a adição das polpas foi utilizado como controle.

\section{PARTE EXPERIMENTAL}

\section{Materiais}

Foram utilizados fécula da mandioca (doado pela Cargill Agrícola S.A.), sacarose e açúcar invertido (doados pelo Açúcar Guarani S.A.) e polpas de manga e acerola adquiridas em supermercados de Salvador, BA.

\section{Preparação dos biofilmes de fécula de mandioca}

Utilizou-se um delineamento estatístico de superfície de resposta, com um modelo de ordem $\left(2^{2}\right)$ contendo 4 pontos axiais, 4 pontos ortogonais e 3 pontos centrais, para definir os diferentes teores das polpas de manga e acerola a serem incorporados aos biofilmes de fécula de mandioca plastificados com açúcar invertido e sacarose, totalizando 11 formulações.

Os valores reais e codificados das polpas de manga $\left(\% \mathrm{~m} / \mathrm{m} ; \mathrm{X}_{1}\right)$ e de acerola $\left(\% \mathrm{~m} / \mathrm{m} ; \mathrm{X}_{2}\right)$ (variáveis independentes), encontram-se na Tabela 1. Os biofilmes foram preparados segundo a técnica casting, que consiste no preparo de uma solução filmogênica, por dissolução em água destilada, das polpas de manga (0 a 20\%) e de acerola (0 a $20 \%)$, da fécula de mandioca $(4 \%, \mathrm{~g} / 100 \mathrm{~g})$ e dos agentes plastificantes sacarose $(0,7 \%, \mathrm{~g} / 100 \mathrm{~g})$ e açúcar invertido $(1,4 \%, \mathrm{~g} / 100 \mathrm{~g})$. Posteriormente, a solução foi aquecida até a temperatura de gelatinização do amido, $70^{\circ} \mathrm{C}$, sob agitação constante. A solução filmogênica foi pesada $(28,06$ a $40,83 \mathrm{~g})$ em de placas de Petri de poliestireno e desidratada em estufa com circulação de $\operatorname{ar}\left(35 \pm 2{ }^{\circ} \mathrm{C}\right)$ por 18 a $20 \mathrm{~h}$. Os biofilmes obtidos foram acondicionados a $60 \%$ umidade relativa (UR) a $23{ }^{\circ} \mathrm{C}$ em dessecadores contendo solução saturada de nitrato de magésio, por 2 dias, antes de serem retirados e caracterizados. ${ }^{15}$

\section{Caracterização dos filmes biodegradáveis}

Os filmes biodegradáveis produzidos a partir das 11 formulações do planejamento estatístico (Tabela 1) com diferentes teores de polpas de manga ( 0 a $20 \%$ ) e de acerola ( 0 a 20\%) foram investigados quanto aos teores de sólidos totais, espessura e taxa de permeabilidade ao vapor de água. Nas formulações F4, máxima incorporação de ambas as polpas (17,1\% de polpa de manga e $17,1 \%$ de polpa de acerola); $\mathrm{F} 1$, mínima incorporação de ambas as polpas (2,9\% de polpa de manga e 2,9\% de polpa de acerola) e F9, mesma incorporação de ambas as polpas ( $10 \%$ de polpa de manga e $10 \%$ de polpa de acerola), além das análises de sólidos totais, espessura e taxa de permeabilidade ao vapor de água, foram realizadas a calorimetria exploratória diferencial (DSC), a avaliação das propriedades mecânicas de resistência à tração e a porcentagem de alongamento.

\section{Teores de sólidos totais}

Os teores de sólidos totais das amostras pré-condicionadas $(60 \%$ $\mathrm{UR}, 23^{\circ} \mathrm{C}$ ) foram determinados após $24 \mathrm{~h}$ de secagem em estufa a $105^{\circ} \mathrm{C} .{ }^{16}$ As análises foram realizadas em triplicata.

\section{Espessura}

A espessura dos filmes pré-acondicionados $\left(60 \%\right.$ UR, $\left.23{ }^{\circ} \mathrm{C}\right)$ foi avaliada através da espessura média resultante de 6 medições em posições aleatórias, por meio de micrômetro digital Mitutoyo de ponta plana (com resolução de $1 \mu \mathrm{m}$ ), em triplicata para cada ponto.

\section{Taxa de permeabilidade ao vapor de água}

A taxa de permeabilidade ao vapor de água dos filmes pré-acondicionados $\left(60 \% \mathrm{UR}, 23^{\circ} \mathrm{C}\right)$ foi medida pelo método gravimétrico modificado. ${ }^{17,18}$ Os filmes foram selados com parafina em células de permeação de alumínio, contendo sílica gel seca (umidade relativa $0 \%$ ) em seu fundo. As células de permeação foram então acondicionadas em dessecadores $\left(25^{\circ} \mathrm{C}\right)$ com umidade relativa controlada por meio de uma solução de $\mathrm{NaCl}$ saturada (UR $=75 \%$ ). As células de permeação com os filmes foram sucessivamente pesadas até o peso da sílica aumentar em 4\%. Como o filme de amido é hidrofóbico, utilizou-se um corpo de prova adicional, filmes de amido sem os aditivos foram colocados em células de permeação sem dessecante

Tabela 1. Valores codificados e reais do delineamento estatístico das variáveis independentes (teores de aditivos) incorporados às formulações de biofilmes de fécula de mandioca. Médias ( \pm desvio padrão) das análises de caracterização das formulações de biofilmes e controle (C)

\begin{tabular}{lccccccc}
\hline & \multicolumn{2}{c}{ Valores codificados } & \multicolumn{2}{c}{ Valores reais $(\% \mathrm{~m} / \mathrm{m})$} & & \multicolumn{2}{c}{ Caracterização } \\
F & $\begin{array}{c}\text { Polpa de } \\
\text { manga }\left(\mathrm{X}_{1}\right)\end{array}$ & $\begin{array}{c}\text { Polpa de } \\
\text { acerola }\left(\mathrm{X}_{2}\right)\end{array}$ & $\begin{array}{c}\text { Polpa de } \\
\text { manga }\end{array}$ & $\begin{array}{c}\text { Polpa de } \\
\text { acerola }\end{array}$ & ST \pm dp & E $\pm \mathrm{dp}$ & TPVA $\pm \mathrm{dp}$ \\
\hline F1 & $-1,00$ & $-1,00$ & 2,90 & 2,90 & $80,24 \pm 0,87^{\mathrm{a}}$ & $0,127 \pm 0,54^{\mathrm{a}}$ & $10,55 \times 10^{-8} \pm 0,87$ \\
F2 & $-1,00$ & 1,00 & 2,90 & 17,1 & $82,03 \pm 1,23$ & $0,130 \pm 0,63^{\mathrm{a}, \mathrm{b}}$ & $7,52 \times 10^{-8} \pm 1,43$ \\
F3 & 1,00 & $-1,00$ & 17,10 & 2,90 & $83,63 \pm 0,64^{\mathrm{c}}$ & $0,141 \pm 0,32^{\mathrm{c}}$ & $7,01 \times 10^{-8} \pm 0,85$ \\
F4 & 1,00 & 1,00 & 17,10 & 17,10 & $84,23 \pm 0,85^{\mathrm{d}}$ & $0,135 \pm 0,87^{\mathrm{c}, \mathrm{d}}$ & $6,62 \times 10^{-8} \pm 1,43^{\mathrm{a}}$ \\
F5 & $-1,41$ & 0,00 & 0,00 & 10,00 & $80,24 \pm 1,18^{\mathrm{a}}$ & $0,127 \pm 0,52^{\mathrm{a}}$ & $7,86 \times 10^{-8} \pm 1,31$ \\
F6 & 1,41 & 0,00 & 20,00 & 10,00 & $84,51 \pm 0,43^{\mathrm{d}}$ & $0,123 \pm 0,84^{\mathrm{a}}$ & $6,74 \times 10^{-8} \pm 0,76^{\mathrm{b}}$ \\
F7 & 0,00 & $-1,41$ & 10,00 & 0,00 & $82,37 \pm 1,45^{\mathrm{b}}$ & $0,125 \pm 0,61^{\mathrm{a}}$ & $8,10 \times 10^{-8} \pm 0,61$ \\
F8 & 0,00 & 1,41 & 10,00 & 20,00 & $83,35 \pm 1,62^{\mathrm{c}}$ & $0,133 \pm 0,20^{\mathrm{b}, \mathrm{d}, \mathrm{e}}$ & $6,70 \times 10^{-8} \pm 0,63^{\mathrm{a}, \mathrm{b}}$ \\
F9 * & 0,00 & 0,00 & 10,00 & 10,00 & $82,88 \pm 0,93^{\mathrm{b}}$ & $0,138 \pm 0,35^{\mathrm{c}, \mathrm{e}}$ & $6,84 \times 10^{-8} \pm 1,21^{\mathrm{b}, \mathrm{c}}$ \\
F10 * & 0,00 & 0,00 & 10,00 & 10,00 & $82,85 \pm 1,72^{\mathrm{b}}$ & $0,136 \pm 0,29^{\mathrm{c}, \mathrm{e}}$ & $6,88 \times 10^{-8} \pm 0,1,06^{\mathrm{c}}$ \\
F11 * & 0,00 & 0,00 & 10,00 & 10,00 & $82,86 \pm 1,15^{\mathrm{b}}$ & $0,140 \pm 0,75^{\mathrm{c}}$ & $6,85 \times 10^{-8} \pm 1,21^{\mathrm{c}}$ \\
C & - & - & - & - & $84,97 \pm 0,31^{\mathrm{d}}$ & $0,128 \pm 0,36^{\mathrm{a}}$ & $9,50 \times 10^{-8} \pm 0,75^{\mathrm{c}}$ \\
\hline
\end{tabular}

C: Controle; F: Formulações; * Pontos centrais; ST: sólidos totais - \%; E: espessura - mm; TPVA: taxa de permeabilidade ao vapor de água - $\mathrm{g} \mathrm{H} \mathrm{H}_{2} \mathrm{O} \mu \mathrm{m} / \mathrm{m}^{2} \mathrm{~h} \mathrm{~mm} \mathrm{H}$. Valores que apresentam a mesma letra, numa mesma coluna, não apresentam diferenças significativas $(\mathrm{p}>0,05)$ pelo teste de Tukey a $95 \%$ de confiança. 
(branco), que permitiu a subtração ou adição da variação de peso do material. As análises foram realizadas em quadriplicata. A taxa de permeabilidade ao vapor d'água (TPVA) foi calculada de acordo com a Equação 1, onde w/t é o coeficiente angular da reta gerada pela perda de peso da amostra em função do tempo; X, é a espessura e A, é a área do filme $\left(0,39 \mathrm{~m}^{2}\right)$.

$$
T P V A=\frac{(w / t) \cdot X}{A}
$$

\section{Propriedades mecânicas}

O percentual de alongamento $(\mathrm{PA})$ e a resistência à tração $(\mathrm{RT})$ foram medidos usando uma máquina universal de ensaio, EMIC Linha DL 20.000 20KN, operando conforme as especificações da ASTM. ${ }^{19,20}$ Foram cortadas tiras $(8 \times 2,5 \mathrm{~cm})$ dos filmes sob as condições de pré-acondicionamento $\left(60 \% \mathrm{UR}, 23^{\circ} \mathrm{C}\right)$ e montadas entre as garras do equipamento. Os valores das espessuras de cada formulação de biofilme necessários para a realização das análises foram medidos em 6 pontos, em posições aleatórias, com um micrômetro digital Mitutoyo de ponta plana. A posição inicial e a velocidade de separação das garras foram fixadas a $50 \mathrm{~mm}$ e $12,5 \mathrm{~mm} / \mathrm{min}$, respectivamente. Sete medidas foram feitas para cada amostra. Como parâmetro de comparação foram utilizadas tiras $(8 \times 2,5 \mathrm{~cm})$ de filmes de fécula de mandioca (sem as polpas) pré-acondicionados $\left(60 \% \mathrm{UR}, 23{ }^{\circ} \mathrm{C}\right)$ nas mesmas condições de análise.

\section{Calorimetria exploratória diferencial}

A análise térmica dos filmes foi realizada usando calorimetria exploratória diferencial (DSC), instrumento universal PerkinElmer, modelo DSC 7. Cerca de 8-10 mg de amostras pré-condicionadas $\left(50 \%\right.$ UR, $25{ }^{\circ} \mathrm{C}$ ) foram hermeticamente seladas em cadinhos de alumínio, para prevenir a evaporação da água durante a varredura. Um cadinho de alumínio vazio serviu como referência. A varredura foi realizada de $-40{ }^{\circ} \mathrm{C}$ a $200{ }^{\circ} \mathrm{C}$, com uma taxa de $10{ }^{\circ} \mathrm{C} /$ min e 10 $\mathrm{mm}$ de $\mathrm{N}_{2} / \min ^{21}$

\section{Análise estatística}

Os dados gerados (variáveis dependentes) foram tratados pela ANOVA e teste de Tukey para identificar se as alterações nos parâmetros avaliados; devido à influência das variáveis independentes (diferentes percentuais de polpas de manga e de acerola) foram significativas ao nível de $95 \%$ de significância.

\section{RESULTADOS E DISCUSSÃO}

As 11 formulações dos filmes biodegradáveis foram investigadas quanto aos teores de sólidos totais, espessura e taxa de permeabilidade ao vapor de água (Tabela 1 ). Nas formulações selecionadas, ou seja, com máximas incorporações das polpas, F4 (17,1\% de manga e $17,1 \%$ de acerola); com incorporações mínimas das polpas, F1 (2,9\% de manga e $2,9 \%$ de acerola) e, com percentuais intermediários de incorporação, F9 (10\% de manga e 10\% de acerola), além dessas análises, foi realizada a calorimetria exploratória diferencial (DSC), bem como a avaliação das propriedades mecânicas de resistência à tração e porcentagem de alongamento. Buscou-se avaliar a influência da adição das polpas de manga e de acerola (fontes de compostos ativos) ${ }^{15}$ sobre estes parâmetros. Para comparação foi utilizado um controle ( $\mathrm{C}$ - biofilme de fécula de mandioca sem os aditivos).

A análise de variância (ANOVA) para os valores de sólidos totais, espessura e taxa de permeabilidade ao vapor de água apresentou valores de $\mathrm{F}_{\text {cal. }}$ para a falta de ajuste do modelo maior do que o $\mathrm{F}_{\text {tab. }}$
$(3,2 ; 0,95=9,55)$ indicando uma falta de ajuste do modelo $(\mathrm{p}>$ $0,05 ; \mathrm{p}>0,20)$.

As variações nos teores de sólidos totais foram significativas entre as diferentes formulações dos filmes, e entre as formulações e o controle ( $\mathrm{p}<0,05)$, com limites de 80,24 e 84,97\%, (Tabela 1).

Os valores de espessura dos filmes variaram de $0,123 \mathrm{~mm}$ para a formulação F6 (20\% de polpa de manga e 10\% de polpa de acerola) a $0,141 \mathrm{~mm}$ para a formulação $\mathrm{F} 3(17,10 \%$ de polpa de manga e 2,90\% de polpa de acerola), enquanto o controle apresentou um valor intermediário $(0,128 \mathrm{~mm})$. Apesar dos biofilmes terem sido elaborados a partir de valores pré-estabelecidos de massa $(2,35 \mathrm{~g}$ de sólidos totais por unidade de filme) para resultar em espessuras semelhantes, foram constatadas diferenças significativas $(p<0,05)$ entre algumas formulações, assim como entre algumas formulações e o controle (Tabela 1). Uma possível justificativa pode estar relacionada à natureza hidrofílica das fibras, que podem provocar processos de sorção com a água e alterar a espessura dos filmes, ${ }^{22}$ uma vez que se constatou correlação linear diretamente proporcional entre os teores de polpa de manga incorporados e os teores de sólidos totais $\left(\mathrm{R}^{2}=\right.$ 0,86). A correlação entre os teores de polpa de manga e as espessuras foi menor $\left(R^{2}=0,05\right)$ (Figura 1A). Estas mesmas correlações não foram constatadas para estes parâmetros e os teores de polpa de acerola incorporados.

Para os biofilmes formulados com a mesma matriz (fécula de mandioca, plastificados com sacarose e açúcar invertido) e adição de diferentes concentrações de extrato de dendê e de azeite de dendê, ${ }^{23}$ e com cacau e extrato de café, ${ }^{24}$ fontes pobres em fibras, as espessuras variaram de 0,125 a $0,160 \mathrm{~mm}$ e de 0,113 a $0,143 \mathrm{~mm}$, respectivamente, sem diferenças significativas entre as diferentes formulações ( $p>0,05)$.

Os valores de permeabilidade das 11 formulações variaram de $6,62 \times 10^{-8}$ a $10,55 \times 10^{-8} \mathrm{~g} \mathrm{H}_{2} \mathrm{O} \mu \mathrm{m} / \mathrm{m}^{2} \mathrm{~h} \mathrm{~mm} \mathrm{Hg}$, enquanto que o controle (C) apresentou um valor de $9,5 \times 10^{-8} \mathrm{~g} \mathrm{H}_{2} \mathrm{O} \mu \mathrm{m} / \mathrm{m}^{2} \mathrm{~h} \mathrm{~mm}$ $\mathrm{Hg}(\mathrm{p}<0,05)$ (Tabela 1). A adição das polpas de manga e de acerola em baixos percentuais na matriz de fécula de mandioca plastificada com sacarose e açúcar invertido resultou em um aumento nos valores de permeabilidade ao vapor de água, enquanto que a incorporação de maiores percentuais reduziu a referida taxa. Estes resultados também podem ser explicados em função da natureza hidrofílica tanto da fécula de mandioca, quanto das fibras oriundas das polpas, principalmente a de manga. ${ }^{13}$ Uma alta taxa de permeabilidade ao vapor de água para filmes de manga, comparados a filmes obtidos de outras frutas já foi relatado. ${ }^{25}$ Muller et al. ${ }^{26}$ atribuíram a redução nos valores da taxa de permeabilidade ao vapor de água a alterações nas estruturas dos filmes de amido de mandioca, causadas pela adição de fibras insolúveis (celulose) que diminuiriam os espaços livres na matriz polimérica, dificultando a passagem do vapor de água através da matriz.

Uma vez detectada a maior influência da polpa de manga sobre os teores de sólidos totais dos filmes, a correlação deste parâmetro com os demais pode ser observada na Figura $1 \mathrm{~B}, \operatorname{com} \mathrm{R}^{2}=0,67$ para os valores da taxa de permeabilidade ao vapor de água e $\mathrm{R}^{2}=0,11$ para a relação com a espessura.

Teoricamente, segundo as leis de difusão de Fick e de sorção de Henry, a taxa de permeabilidade ao vapor de água varia de modo inverso com a espessura do filme. ${ }^{27,28}$ Apesar do baixo coeficiente de determinação $\left(R^{2}=0,22\right)$ em função da dispersão dos pontos experimentais, constatou-se que a variação da taxa de permeabilidade ao vapor de água foi inversamente proporcional à espessura, obedecendo as referidas leis (Figura 1C).

A incorporação de fibras à matriz de fécula de mandioca pode aumentar a tortuosidade do material, resultando um processo de difusão menor e, então, numa menor taxa de permeabilidade do vapor 

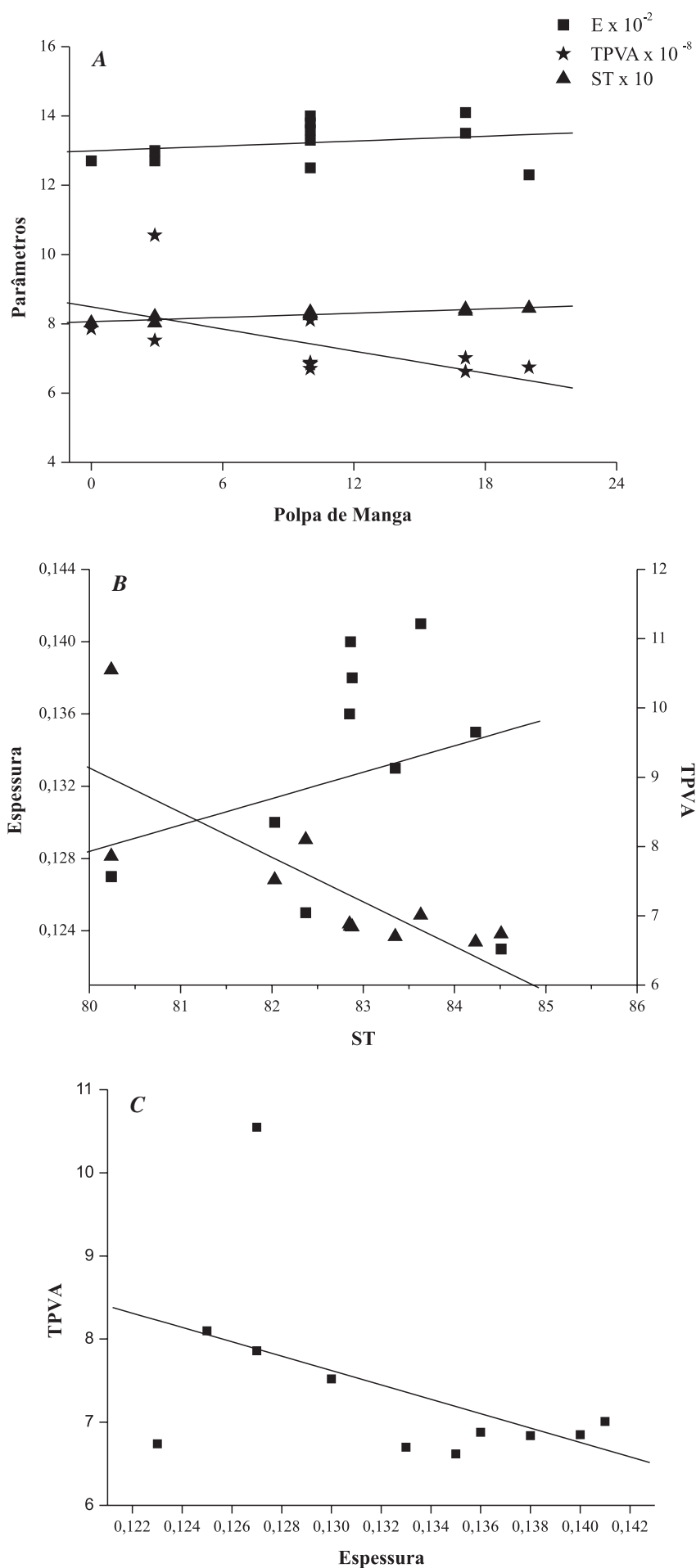

Figura 1. A - Correlação entre a variável independente (polpa de manga) e as variáveis dependentes (ST: Sólidos Totais em \%; E: Espessura em mm; TPVA: Taxa de Permeabilidade ao Vapor de Água em $10^{-8} \mathrm{~g} \mathrm{H}_{2} \mathrm{O} \mu \mathrm{m} / \mathrm{m}^{2} \mathrm{~h} \mathrm{~mm} \mathrm{H}$ ). $B$-Correlação entre os teores de ST e a E. C-Correlação entre a TPVA e a $E$

de água (Tabela 1 e Figura 1). Há relato de que a interação de fibras de celulose com componentes de purê de manga (principalmente pectinas, amido e/ou derivados de celulose) pode atuar como barreira ao vapor de água. ${ }^{9}$ Portanto, os sítios hidrofílicos das fibras incorporadas (principalmente da polpa de manga) podem interagir com os grupos hidroxílicos do amido de mandioca através de ligações de hidrogênio.

A diminuição linear da taxa de permeabilidade ao vapor de água em função do aumento da espessura também foi observada por
Martin-Polo et al., ${ }^{27}$ em filmes de celofane revestidos com parafina $(0,050$ a $0,140 \mathrm{~mm})$ e por Sobral, ${ }^{29}$ em filmes de proteínas miofibrilares $(0,017$ a $0,102 \mathrm{~mm})$. Segundo Park e Chinnan, ${ }^{30}$ as razões desse comportamento estão relacionadas a possíveis mudanças estruturais na matriz do filme.

Os valores dos percentuais de alongamento das formulações com teores máximos, mínimos e centrais de incorporação de polpas de manga e de acerola, assim como o controle, estão apresentados na Tabela 2. A formulação com a menor incorporação de polpas de manga e de acerola (F1) apresentou a menor porcentagem de alongamento $(68,67 \%)$, entretanto, a formulação com o maior teor de ambas as polpas (F4) apresentou o maior percentual (72,45\%) (Tabela 2). Ou seja, quanto maior a adição de polpas de manga e de acerola maior o percentual de alongamento. Quando comparadas com o controle, as três formulações apresentaram menores percentuais de alongamento, demonstrando que a incorporação de ambas as polpas promoveu uma diminuição do percentual de alongamento dos biofilmes ( $\mathrm{p}<$ $0,05)$. Resultados semelhantes foram obtidos ao incorporar derivados de dendê em biofilmes de fécula de mandioca, ou seja, o controle apresentou um maior percentual de alongamento quando comparado às formulações com os aditivos..$^{23}$

Observa-se também que a incorporação dos aditivos nas maiores concentrações (F4, 17,1\% m/m de polpa de manga e de acerola) resultou em uma diminuição significativa $(\mathrm{p}<0,05)$ da resistência à tração, quando comparada com as formulações com menores concentrações de aditivos (F9, 10\% m/m de polpa de manga e de acerola; e F1, 2,9\% $\mathrm{m} / \mathrm{m}$ de polpa de manga e de acerola). Quando comparadas com o controle $\mathrm{C}$, as três formulações (F1, F4 e F9) apresentaram valores significativamente inferiores $(\mathrm{p}<0,05)$, (Tabela 2). As relações entre os teores de fibras incorporados aos filmes e os resultados da resistência à tração e percentual de alongamento obtidos contrariam as afirmações de $\mathrm{Ma}^{31}$ e Lu et al., ${ }^{32}$ sugerindo que a diminuição da rigidez dos filmes causada pela incorporação das polpas pode estar associada a uma fraca compatibilidade entre as macromoléculas das fibras das polpas e as do amido.

Apesar das variáveis independentes não terem influência significativa sobre as variáveis dependentes (ANOVA até $\mathrm{p}>0,20$ ), foram constatadas correlações lineares e proporcionais entre os teores de polpa de manga e os sólidos totais e entre os teores de polpa e o percentual de alongamento $\left(\mathrm{R}^{2}=0,92\right)$, assim como correlação inversamente proporcional entre os teores de polpa de manga e a resistência à tração $\left(\mathrm{R}^{2}=0,89\right)$ e entre os teores de sólidos totais e a resistência à tração $\left(R^{2}=0,92\right)$.

Isto provavelmente se deve ao aumento de matéria seca (sólidos totais) por superfície, isto é, ao aumento do número de cadeias de fibras por superfície, o que pode resultar em alteração das interações inter e intramoleculares.

Efeitos similares foram obtidos para a incorporação de baixas concentrações de cacau e café a filmes de fécula de mandioca, resultando em uma menor resistência à tração quando comparada a concentrações maiores. ${ }^{24}$ Filmes de purê de manga e de outras frutas apresentam resistência à tração e percentual de alongamento reduzidos quando comparados a filmes elaborados com amidos de frutas plastificados com glicerol. ${ }^{25}$ Em biofilmes de fécula de mandioca contendo extrato de espinafre também foi verificado um aumento no percentual de alongamento, enquanto que os biofilmes contendo extrato de uva apresentaram uma redução neste parâmetro. Entretanto, apesar dos relatos contraditórios, todos os biofilmes apresentaram uma redução na resistência à tração quando comparados ao controle..$^{25-33}$

Os valores de resistência à tração encontrados no presente estudo são inferiores aos relatados para filmes sintéticos de polietileno de baixa densidade (PEBD), que variam de 6,9 a $16 \mathrm{MPa} .{ }^{34}$ 
Tabela 2. Parâmetros mecânicos e térmicos (DSC) das formulações dos biofilmes selecionadas e do controle. RT (resistência à tração; PA (porcentagem de alongamento); Tgs (temperatura de transição vítrea); $\Delta$ Hs (variação de entalpia); F1 (2,9\% de polpa de manga e 2,9 \%de polpa de acerola); F4 (17,1\% de polpa de manga e 17,1\% de polpa de acerola); F9 (10\% de polpa de manga e 10\% de polpa de acerola); e C (biofilme sem adição das polpas de manga e de acerola)

\begin{tabular}{|c|c|c|c|c|c|c|}
\hline \multirow{2}{*}{$\mathrm{F}^{*}$} & \multirow{2}{*}{$\begin{array}{c}\mathrm{PA} \pm \mathrm{dp} \\
(\%)\end{array}$} & \multirow{2}{*}{$\begin{array}{c}\mathrm{RT} \pm \mathrm{dp} \\
(\mathrm{MPa})\end{array}$} & \multirow[t]{2}{*}{$\operatorname{Tg}\left({ }^{\circ} \mathrm{C}\right)$} & \multicolumn{3}{|c|}{ Onset e picos dos eventos exotérmicos em ${ }^{\circ} \mathrm{C}\left(\Delta \mathrm{Hs}, \mathrm{J} / \mathrm{g}^{-1}\right)$} \\
\hline & & & & 1 & 2 & 3 \\
\hline $\mathrm{F} 1$ & $68,67^{\mathrm{a}} \pm 2,34$ & $4,51^{\mathrm{c}} \pm 1,23$ & $-16,80$ & & 120,65 e $128,44(1,72)$ & 139,67 e $141,32(4,21)$ \\
\hline $\mathrm{F} 4$ & $72,45^{\mathrm{c}} \pm 1,56$ & $1,66^{\mathrm{a}} \pm 1,45$ & - & - & 114,92 e $124,96(174,39)$ & - \\
\hline F9 & $69,58^{\mathrm{b}} \pm 2,56$ & $1,85^{\mathrm{b}} \pm 2,33$ & $-16,74$ & 82,63 e $94,15(19,97)$ & 125,11 e $126,20(20,76)$ & - \\
\hline $\mathrm{C}$ & $76,28^{\mathrm{d}} \pm 2,51$ & $6,49^{\mathrm{d}} \pm 1,87$ & $-16,80$ & - & 130,16 e $132,15(1,66)$ & 143,26 e $145,05(4,64)$ \\
\hline
\end{tabular}

*Formulações. Valores com a mesma letra, numa mesma coluna, não apresentam diferenças significativas ( $p>0,05)$ pelo teste de Tukey a $95 \%$ de confiança.

As curvas DSC $\left(-40\right.$ a $\left.200{ }^{\circ} \mathrm{C}\right)$ das formulações controle, com adição máxima, intermediária e mínima de polpas mostram que os eventos são complexos, indicando uma transição de segunda ordem, com Tgs entre $-16,74$ a $-16,80{ }^{\circ} \mathrm{C}$, e outros eventos de primeira ordem, todos exotérmicos, podendo representar fenômenos físicos, como adsorção ou cristalização, e/ou químicos, como decomposição ou degradação dos componentes dos biofilmes (Tabela 2). Valores negativos de Tgs sugerem uma estabilidade relativamente baixa do material, por causa da alta mobilidade molecular (e, consequentemente, alta reatividade) dos seus componentes. Por outro lado, um valor de Tg baixo implica também numa boa flexibilidade do filme em temperaturas de refrigeração e/ou congelamento, o que pode ser uma vantagem quando se considera o potencial de aplicação como material de embalagem de alimentos. ${ }^{35}$

O biofilme controle, sem adição das polpas de manga e de acerola, apresentou dois eventos exotérmicos. A formulação F1 (com mínima incorporação dos aditivos, $2,9 \%$ de polpa de manga e 2,9\% de polpa de acerola) apresentou eventos térmicos similares aos do controle, com pequenas mudanças nas temperaturas máximas, indicando poucas alterações. A formulação F9 (com valores intermediários dos aditivos, $10 \%$ de polpa de manga e $10 \%$ de polpa de acerola) apresentou dois eventos exotérmicos, sendo um deles em temperatura inferior e bastante diferente do controle. A formulação F4 (com máxima incorporação dos aditivos, $17 \%$ de polpa de manga e $17 \%$ de polpa de acerola) apresentou apenas um evento. Ou seja, a máxima incorporação das polpas de manga e de acerola aos filmes diminuiu o número de eventos térmicos, podendo indicar uma baixa separação de fases, provavelmente porque os aditivos nessas concentrações estão mais envolvidos pela matriz, formando um material mais homogêneo. Vale considerar que estas alterações nas propriedades térmicas podem ser devidas não somente à estrutura molecular das fibras, mas a outros compostos oriundos das polpas que, individualmente ou em conjunto, exercem um efeito maior sobre a mobilidade da cadeia global do filme de amido, justificado também pelo aumento no percentual de elongação obtido em F4.

Os valores de $\Delta \mathrm{Hs}$ dos eventos térmicos das formulações $\mathrm{F} 1$, F4 e F9 apresentaram diferenças quando comparados entre si e ao controle. Considerando que o primeiro evento exotérmico a 94,15 ${ }^{\circ} \mathrm{C}$ foi apresentado somente pela $\mathrm{F} 9$ e que os $\Delta \mathrm{Hs}$ do terceiro evento (entre 140 e $145^{\circ} \mathrm{C}, \mathrm{F} 1$ e controle) são similares, o único evento apresentado por todas as formulações (entre 124,96 e $132,15^{\circ} \mathrm{C}$ ) foi o que mostrou uma maior variação da área, com entalpia entre 1,66 a 174,39 J g- ${ }^{1}$, que aumenta proporcionalmente com a incorporação das polpas aos biofilmes, (Tabela 2). Isto pode ressaltar a hipótese deste evento ser decorrente de um processo físico de cristalização ou semicristalização do material, sendo necessária maior energia à medida que aumenta o teor de fibras oriundas principalmente da polpa de manga incorporada ao amido. $\mathrm{O}$ aumento da entalpia necessária ao evento sugere um aumento nas características cristalinas do material, o que poderia justificar a menor resistência à tração de F4, portanto, as microfibrilas incorporadas podem estar agindo como agentes de nucleação para formação de uma fase cristalina ou semicristalina. O conteúdo de amilose da fécula de mandioca varia de 17 a $24 \%$, entretanto a sua cristalinidade está associada com a presença da amilopectina. ${ }^{36-38}$ Portanto, pode-se supor que no presente estudo a cristalinidade dos biofilmes foi intensificada à medida que se aumentou o teor de fibra incorporada ao biofilme. Portanto, a energia necessária para a desorganização da ordem molecular difere de acordo com a formulação do biofilme, sendo maior na formulação com máxima quantidade de polpas de manga e acerola incorporadas (F4) $\left(\Delta \mathrm{H}=174,39 \mathrm{~J} \mathrm{~g} \mathrm{~g}^{-1}\right)$, seguida pela formulação $\mathrm{F} 9$, com $10 \%$ de polpa de manga e $10 \%$ de polpa de acerola $\left(\Delta \mathrm{H}=20,76 \mathrm{~J} \mathrm{~g}^{-1}\right)$, e pela $\mathrm{F} 1$ que possui os menores teores de polpas adicionadas $\left(\Delta \mathrm{H}=1,72 \mathrm{~J} \mathrm{~g}^{-1}\right)$ que é aproximadamente igual ao controle $\left(\Delta \mathrm{H}=1,66 \mathrm{~J} \mathrm{~g}^{-1}\right)$, (Tabela 2).

$\mathrm{O}$ intervalo de gelatinização da fécula de mandioca ocorre entre 58 e $70^{\circ} \mathrm{C},{ }^{39}$ considerando que nenhuma das formulações (F1, F4, F9 e controle) apresentou evento endotérmico largo entre 15 a 145 ${ }^{\circ} \mathrm{C}\left(\Delta \mathrm{H}=113 \mathrm{~J} \mathrm{~g}^{-1}\right),{ }^{40}$ comprova-se que todo o amido da fécula de mandioca foi pré-gelatinizado durante o processamento empregado na elaboração dos filmes. A gelatinização é definida como uma transição ordem-desordem que envolve a ruptura da organização molecular dentro dos grânulos de amido, sob aquecimento e na presença de plastificantes. O plastificante atua rompendo as ligações de hidrogênio amido-amido entre hélices de amilopectina, promovendo ligações de hidrogênio amido-plastificante simultaneamente. ${ }^{40}$

Portanto, a incorporação de diferentes teores de polpas de manga e de acerola como aditivos em biofilmes de fécula de mandioca, plastificados com sacarose e açúcar invertido, altera significantemente as propriedades mecânicas, térmicas, de elongação e a permeabilidade ao vapor de água dos filmes, quando comparados com o filme elaborado somente com a matriz e os mesmos plastificantes. Dependendo dos teores de incorporação destas polpas à matriz de fécula de mandioca, pode-se obter uma nova matriz devido a: interações da água com as fibras, contribuindo para uma reorganização molecular do material da nova matriz; interações entre os componentes da matriz e das polpas, resultando em um aumento na mobilidade da matriz. Por outro lado, as fibras das polpas, por si só, podem promover um aumento da fase cristalina ou semicristalina e, consequentemente, uma mobilidade restrita da nova matriz.

Filmes comestíveis produzidos pela incorporação de polpas de manga e de acerola, além de proporcionarem cor e alta ação antioxidante devido aos pigmentos presentes nas polpas, ${ }^{15}$ resultam em filmes com flavor característico da(s) fruta(s) devido à incorporação simultânea de compostos voláteis, podendo combinar também propriedades térmicas, mecânicas e de barreira ao vapor de água.

Estes resultados revelam que, juntas, as polpas de manga e de acerola apresentam um bom potencial para serem incorporadas em até $34,2 \% \mathrm{~m} / \mathrm{m}$ em filmes biodegradáveis de amido de mandioca, que podem ser aplicados como revestimento de alimentos. 


\section{CONCLUSÕES}

Os resultados apresentados indicam que a incorporação de polpas de manga e de acerola a biofilmes de fécula de mandioca, plastificados com sacarose e açúcar invertido, afeta as propriedades mecânicas, térmicas, de elongação e de permeabilidade ao vapor de água da matriz. Em baixas concentrações os aditivos promovem um aumento da resistência à tração e diminuição da porcentagem de alongamento, porém promovem um aumento na permeabilidade ao vapor de água. $\mathrm{O}$ aumento da concentração dos aditivos provoca o inverso, uma diminuição na resistência à tração, um aumento na porcentagem de alongamento e uma diminuição na permeabilidade ao vapor de água, comparando ao controle. Quando comparadas ao controle todas as formulações apresentaram diminuição da resistência à tração e da porcentagem de alongamento, além de diminuição no número de temperaturas de transição vítreas. Portanto, biofilmes formulados a partir de fécula de mandioca plastificados com sacarose e açúcar invertido podem ter suas propriedades significativamente alteradas pela incorporação de polpas de manga e de acerola. Trabalhos futuros poderão avaliar o efeito da incorporação de polpas de manga e de acerola em outras matrizes biodegradáveis ou em materiais poliméricos convencionais, aumentando a possibilidade de aplicações.

\section{REFERÊNCIAS}

1. Tharanathan, R. N.; Trends Food Sci. Technol. 2003, 14, 71.

2. Parra, D. F.; TadiniI, C. C.; Ponce, E. P.; Lugão, A. B.; Carbohydr. Polym. 2004, 58, 475.

3. Ludueña, L. N.; Alvarez, A. V.; Vazquez, A.; Mater. Sci. Eng. 2007, 460, 121.

4. Rooney, M. L.; Active Food Packaging 1995, 1, 3.

5. Brody, A. L.; Food Technology 2001, 55, 9.

6. Lourdin, D.; Coignard, L.; Bizot, H.; Colonna, P.; Polymers 1997, 38, 5401.

7. Veiga-Santos, P.; Oliveira, L. M.; Cereda, M. P.; Scamparini, A. R. P.; Food Chem. 2007, 103, 255.

8. Larrauri, J. A.; Rupérez, P.; Borroto, B.; Saura-Calixto, F.; Lebensmittel Wissenschaft und Technologie 1996, 29, 729.

9. Sanchez-Garcia, M. D.; Gimenez, E.; Lagaron, J. M.; Carbohydr. Polym. 2008, 71, 235.

10. Azizi Samir, M. A. S.; Alloin, F.; Dufresne, A.; Biomacromolecules 2005, 6, 612.

11. Dalmas, F.; Cavaill'e, J. Y.; Gauthier, C.; Chazeau, L.; Dendievel, R.; Composites Science and Technology 2007, 67, 829.

12. Dufresne, A.; Vignon, M. R.; Biomacromolecules 1998, 31, 2693.
13. Avérous, L.; Fringant, C.; Moro, L.; Polymer 2001, 42, 6565.

14. Curvelo, A. A. S.; Carvalho, A. J. F.; Agnelli, J. A. M.; Polymer 2001, $45,183$.

15. Souza, C. O.; Silva, L. T.; Silva, J. R.; Lopez, J. A.; Veiga-Santos, P.; Druzian, J. I.; J. Agric. Food Chem. 2011, 59, 2248.

16. Pouplin, M.; Redl, A.; Gontard, N.; J. Agric. Food Chem. 1999, 47, 538.

17. American Society for Testing and Materials; Standard practice for maintaining constant relative humidity by means of aqueous solution, ASTM E104-85, 2001.

18. Gontard, N.; Guilbert, S.; Cuq, J.L.; J. Food Sci. 1992, 57, 190.

19. American Society for Testing and Materials; Standard Test Method for tensile properties of thin plastic sheeting, ASTM D882-00, 2001.

20. Veiga-Santos, P.; Oliveira, L. M.; Cereda, M. P.; Alves, A. J.; Scamparini, A. R. P.; Food Hydrocolloids 2005, 19, 341.

21. Sobral, P. J. A.; Thermochim. Acta 2001, 376, 83.

22. Stading, M.; Rindlav-Westling, A.; Gatenholm, P.; Carbohydr. Polym. 2001, 45, 209.

23. Grisi, C.; Dissertação de Mestrado, Universidade Federal da Bahia, Brasil, 2008.

24. Silva, L. T.; Dissertação de Mestrado, Universidade Federal da Bahia, Brasil, 2009.

25. Sothornvit, R.; Rodsamran, P.; Postharvest Biology and Technology 2008, 47, 407.

26. Muller, C.; Yamashita, F.; Laurindo, J. B.; Food Hydrocolloids 2009, 23, 1328.

27. Martin-Polo, M.; Mauguin, C.; Voilley, A.; J. Agric. Food Chem. 1992, 40, 407.

28. Cuq, B.; Gontard, N.; Cuq, J. L.; Guilbert, S.; J. Food Sci. 1996, 61, 580.

29. Sobral, P. J. A.; Pesq. Agropec. Bras. 2000, 35, 1251.

30. Park, H. J.; Chinnan, M. S.; J. Food Eng. 1995, 25, 497.

31. Ma, X.; Yu, J.; Kennedy, J. F.; Carbohydr. Polym. 2005, 62, 19.

32. Lu, Y.; Weng, L.; Cao, X.; Carbohydr. Polym. 2006, 63, 198.

33. Attarian, A. C.; Veiga-Santos, P.; Ditchfield, C.; Parra, D. F.; Lugão, A. B.; Tadini, C. C.; Resumos do VI International symposium on future of food engineering, Warsaw, Poland, 2006.

34. Coutinho, F. M. B.; Mello, I. L.; Maria, L. C. S.; Polímeros 2003, 13, 1.

35. Rogers, C. E.; Permeation of gases and vapors in polymer, $4^{\text {th }}$ ed., Elsevier Appl. Sci. Publ.: London, 1985.

36. Defloor, I.; Dehing, I.; Delcour, J. A.; Starke 1998, 50, 58.

37. Yu, L.; Christie, G.; Carbohydr. Polym. 2001, 46, 179.

38. Zhang, G.; Ao, Z.; Hamaker B. R.; Biomacromolecules 2006, 7, 3259.

39. Bobbio, F. O.; Bobbio, P. A.; Introdução à Química de Alimentos, $2^{\mathrm{a}}$ ed., Varela: São Paulo, 1995.

40. Schlemmer, D.; Sales, M. J. A.; Resck, I. S.; Ciência e Tecnologia de Alimentos 2010, 20, 6. 